\title{
CINÉTICA DE ABSORÇÃO DO CÁLCIO DO LEITE E DA CASCA DE OVO NO INTESTINO DELGADO DE RATOS 1
}

\author{
ALICE KATSUKO OGUIDO2 \\ REBECA CARLOTA DE ANGELIS ${ }^{3}$ \\ INÊS FUMIKO UBUKATA YADA4
}

OGUIDO, Alice Katsuko; DE ANGELIS, Rebeca Carlota. Cinética da absorção do cálcio do leite e da casca de ovo no intestino delgado de ratos. Semina: Ci. Agr., Londrina, v. 16, n. 1, p. 7-13, mar. 1995.

RESUMO: O presente trabalho teve por objetivo determinar os parâmetros absortivos e parâmetros cinéticos de cálcio no leite e na casca de ovo, através de técnica da alça ligada "in situ" e "in vivo". Para a determinação dos parâmetros cinéticos foram utilizados os métodos dos mínimos quadrados, regressão inversa ponderada. Os parâmetros absortivos determinados foram melhores na casca de ovo quando comparados com leite e $\mathrm{CaCl}_{2}$, porém, os parâmetros cinéticos foram melhores no leite.

PALAVRAS-CHAVE: Absorção de cálcio; cinética; leite; casca de ovo.

\section{INTRODUÇĀO}

O crescente reconhecimento da importância nutricional de cálcio para desenvolver um esqueleto do tamanho e vigor que se aproxima do potencial genético torna o estudo da absorção intestinal de cálcio pertinente e relevante.

A adequada ingestão de minerais é determinada pela eficiência na absorção a partir de diferentes tipos de alimentos, de dietas e de matérias alimentícias, e sob diferentes condições e estado fisiológico.

Segundo ROSEMBERG \& SOLOMONS (1982), para uma análise de absorção de minerais, devem-ser levar em consideração os fatores que influenciam a sua biodisponibilidade, como, por exemplo, a forma química do mineral, a valência, a interação no lúmen com protelnas, peptídios, amino ácidos, ânions e outros metais (ALLEN, 1982).

Vårios componentes dos alimentos ou dietas interferem na absorção de cálcio: a vitamina D, proteínas, lactose alumentam a absorção, enquanto que substâncias como o ácido fítico, fibras de dieta, ácido oxálico e gorduras prejudicam a sua absorção (MCBEAN \& SPECKMANN, 1974).

MARSHALL (1976) e TOVERUD \& DOSTAL (1986) traduziram a absorção de cálcio segundo um modelo matemático de regressão linear $Y=A+B x$, no qual a interesecção $A$ com a ordenada é uma estimativa do transporte máximo pelo processo ativo, e a inclinação
B representa a contribuição do componente passivo. A relativa contribuição da absorção ativa é maior em baixas concentrações de cálcio luminal (abaixo de $9 \mathrm{mM}$ ). O componente passivo é representado pela linha reta e o componente ativo é a diferença desta linha e o total de cálcio absorvido.

Um artigo de revisão realizado por BRONNER et al. (1986) traz alguns parâmetros cinéticos de $V \max =22$ umol.h-1/g $(\mathrm{p} / \mathrm{p}-1)$, e $\mathrm{K}=3,9 \mathrm{mM}$ e a taxa do componente não saturável de 0,16 que poderão ser utilizados para predizer os resultados da absorção de cálcio.

As características de transporte de cálcio na absorção intestinal têm sido extensivamente estudadas (WALLING \& ROTHMAN, 1969; ARMBRECHT et alı, 1980; WASSERMAN, 1981; WASSERMAN \& FULLMER, 1983; PANSU et al., 1993) em animais adultos há muito tempo, entretanto, as caracteristicas cinéticas de transporte de cálcio em animais jovens têm sido estudadas a partir da década de 80.

Por isso, procurou-se explorar a bio-utilização de cálcio proveniente de diferentes alimentos e obter mais informaçōes sobre os parâmetros cinéticos de absorção de cálcio intestinal em ratos jovens.

\section{2 - MATERIAL E ME̊TOdOS}

\section{1 - ANIMAIS}

Foram utilizados ratos machos (Rattus norvegi-

\footnotetext{
1 - Parte da Tese de Doutorado, apresentada ao Departamento de Alimentos e Nutrição Experimental da F.C.F. da USP. Órgãos Financiadores - CONCITEC/Paraná.

2 - Departamento de Ciências Fisiológicas, Centro de Ciências Biológicas, Universidade Estadual de Londrina, Londrina - PR, Brasil, Caixa Postal 6001, CEP 86051-970.

3 - Departamento de Fisiologia, Centro de Nutrição, USP.

4 - Departamento de Biometria, IAPAR, Londrina - PR.
} 
cus, var. albinus) da linhagem Wistar, com 1 a 1,5 meses de idade com cerca de $100+8 \mathrm{~g}$ de peso corporal. Esses animais foram obtidos do Biotério de Ciências Biomédicas da USP e receberam ação.proveniente da Nuvital Nutrientes Ltda. até um dia antes do experimento. $O$ conteúdo de cálcio determinado nessa ração foi de $1 \mathrm{~g} / 100 \mathrm{~g}$ de ração.

As fontes de cálcio escolhidas foram o leite em pó e a casca de ovo. A casca de ovo que não é considerada alimento, contém grandes quantidades de cálcio, $31,56 \mathrm{~g}$ em $100 \mathrm{~g}$, e tem sido cogitada para servir de fonte de cálcio para suplementação de dietas ou alimentos.

\section{2 - PREPARAÇÃO DAS SOLUÇÕES DE CÁLCIO}

Leite: utilizou-se o leite em pó (marca Ninho, Nestlé) obtido do comércio local. Tomaram-se $55,55 \mathrm{~g}$ de leite em pó e diluiu-se em solução fisiológica. Tratou-se, em seguida com ácido clorídrico e neutralizou-se com hidróxido de sódio. As 6 soluções de cálcio do leite preparadas a partir da solução anterior continham 66,$25 ; 75,63 ; 91,86$; 116,86; 165,00; e 490,25 ugCa/ml.

Casca de ovo: partiu-se de $100 \mathrm{~g}$ de casca de ovo moído no moinho de bolas e seco em estufa a $105^{\circ} \mathrm{C}$. Adicionou-se ácido clorídrico concentrado, o que permitiu a dissolução total da casca de ovo e, em seguida neutralizou-se com hidróxido de sódio. A partir dessa solução, 6 soluções foram preparadas com as seguintes concentrações de cálcio: 61,$25 ; 74,00 ; 90,00 ; 118,75 ; 181,00$; e $410,00 \mathrm{ugCa} / \mathrm{ml}$.

$\mathrm{CaCl}_{2}$ : partiu-se de $1,47 \mathrm{~g}$ de $\mathrm{CaCl}_{2} 2 \mathrm{H}_{2} \mathrm{O}$ diluído em solução fisiológica. Preparou-se, então 6 outras soluções, cujas concentrações foram de: 48,00; 74,00; 105,00; 134,00; 162,00 e 204,00 ugCa/ml. Preparou-se também uma solução cuja concentração determinada foi de 1.340 $\mathrm{ugCa} / \mathrm{ml}$.

\section{3 - PROCEDIMENTO EXPERIMENTAL}

Utilizou-se a técnica da alça ligada "in situ" e "in vivo" (PANSU et al., 1976).

Os animais foram deixados em jejum cerca de 24 horas antes da cirurgia e anestesiados com éter. Fez-se um corte longitudinal no abdomen para a exposição do intestino delgado e colocou-se uma ligadura a $1,5 \mathrm{~cm}$ do piloro e outro a $10 \mathrm{~cm}$ da válvula fleocecal.

Com uma seringa calibrada foram introduzidos 2 $\mathrm{ml}$ de cada uma das soluções em teste. Após 30 minutos, - conteúdo intestinal foi recolhido com uma lavagem com solução fisiológica.

As amostras foram tratadas com a mistura de ácido nítrico e perclórico (proporção de 3:1). Após as devidas diluições, o conteưdo total de cálcio foi determinado no espectrofotômetro de absorção atômica pelo método de digestão ůmida. (LOTT et al., 1956).
2.4 - DELINEAMENTO ESTATÍSTICO

Partindo das premissas de que:

a) a absorção de cálcio no intestino delgado de ratos é uma soma de dois processos ativos e passivo (PANSU et al., 1983);

b) a absorção de cálcio, em elevadas concentrações, é diretamente proporcional a sua concentração (WASSERMAN \& TAYLOR, 1969);

c) o intervalo ideal de concentração para fixar os parâmetros cinéticos varia de 0,3 a $28,7 \mathrm{mM}$ de cálcio, ou seja, de 12 a $748 \mathrm{ugCa} / \mathrm{ml}$ (BRONNER et alı, 1986);

d) são necessários pelo menos 6 niveis de concentração de cálcio para uma estimativa razoável dos parâmetros cinéticos Vmax, $\mathrm{Km}$ e P (PAPWORTH \& PATRICK, 1970).

Foram considerados fontes de cálcio de alimentos 0 leite e a casca de ovo e $\mathrm{CaCl}_{2}$ como padrão.

Para cada. fonte, ajustou-se a curva referente à parte ativa com 6 pontos, subtraídos da regressão linear (slope) com no mínimo 2 pontos (2 do padrão) e o referente ao ponto de concentração mais elevada da fonte em questão.

A determinação dos parâmetros cinéticos seguiu o seguinte roteiro:

1. Curva total de absorção

Plotou-se a absorção versus concentração da solução

2. Ajuste linear da parte de elevada concentração de cálcio com o padräo $\mathrm{CaCl}_{2}$.

Determinaram-se os valores preditos de $\mathrm{P}$.

3. Determinação da absorção ativa nos 6 niveis de concentração

$A t=A a t+A p a s$

Aat $=A t-$ Apas

onde: $A t=$ absorção total

Aat $=$ absorção ativa

Apas $=$ absorção passiva

4. Ajuste da equação de Michaelis e Menten:

$A=\frac{V \max \left(\mathrm{Ca}^{2+}\right)}{K m+\left(\mathrm{Ca}^{2+}\right)}$

pelo método dos mínimos quadrados (BUCLON et ala, 1979 e CLELAND, 1967).

5. Determinação dos valores de $V \max$ e $\mathrm{Km}$ para cada uma das fontes através da regressäo inversa ponderada:

$$
\begin{aligned}
& \frac{1}{A}=\frac{K m+\left(C a^{2+}\right)}{V \max \cdot\left(C a^{2+}\right)}=\frac{1}{V \max }+\frac{K m}{V \max } \cdot \frac{1}{\left(C a^{2+}\right)} \\
& Y=a+b x
\end{aligned}
$$



Portanto:
$Y=\frac{1}{A}$
$x=\frac{1}{\left(\mathrm{Ca}^{2+}\right)}$
$\mathrm{V} \max =\frac{1}{\mathrm{a}}$
e
$\mathrm{Km}=\frac{\mathrm{b}}{\mathrm{a}}=\mathrm{b} \cdot \mathrm{V} \max$

A variação de $\mathrm{Km}$ neste trabalho foi de 0,20 a 5,00 e a região do processo saturável foi de 0,20 a 1,46.

\section{3 - RESUltAdOS E Discussāo}

A quantidade total de cálcio absorvido em 30 minutos é igual a:

$A=1-(D-S)$, onde

$A=$ quantidade total de cálcio absorvido em 30 minutos.

1 = quantidade de cálcio introduzido na alça intestinal, ou seja, o valor da concentração da solução de cálcio do alimento em questão multiplicado por 2.

$\mathrm{D}$ = quantidade de cálcio determinado no conteůdo intestinal.

$\mathrm{S}=$ quantidade de cálcio secretado, ou seja, determinados com os ensaios com solução fisiológica.

O S determinado foi de 60,76 ugCa para o leite, 72,38 ugCa para a casca de ovo e 60,00 ugCa para $\mathrm{CaCl}_{2}$.

$\mathrm{Na}$ Tabela 1 consta a média dos resultados da quantidade de cálcio determinados no conteúdo intestinal de ratos tratados com leite, casca de ovo e $\mathrm{CaCl}_{2}$ nos diferentes niveis testados.

Pode-se notar, através da Tabela 2, que a quantidade total de cálcio absorvida aumentou com a elevação das concentrações das soluções de cálcio nos alimentos testados a $_{\text {. }}$ que não podemos afirmar em relação à porcentagem de absorção de cálcio (Tabela 3) que decresceu com o aumento das concentrações de cálcio das soluções de alimentos introduzidas na alça intestinal.

Assim, os dados sugerem uma relação inversa entre a absorção intestinal e a quantidade presente no lúmen, fato esse análogo ao verificado por MALM (1958) e HEANEY et al. (1975), que demonstraram em humanos que a fração de cálcio absorvida da dieta é inversamente proporcional à quantidade ingerida.

As menores porcentagens de absorção ocorreu com $\mathrm{CaCl}_{2}$ em todos os niveis estudados comparados com o leite e casca de ovo (Tabela 3 ). Esses resultados estão compativeis com o experimento de RECHER et al.
(1988), no qual os autores testaram a absorção de cálcio de 6 diferentes fontes de cálcio e encontraram que o cálcio absorvido de sal foi menor dentre as fontes de alimentos testados, como aconteceu neste trabalho.

Interessante notar que, nos níveis estudados, as prcentagens de absorção foram maiores na casca de ovo quando comparados com as de leite. Resultados esses não esperados, uma vez que o leite contém fatores auxiliadores de absorção como proteínas e lactose.

A taxa de absorção de cálcio do leite, da casca de ovo e de $\mathrm{CaCl}_{2}$ aumentou progressivamente à miedida que se aumentaram as concentrações de cálcio luminal (Tabela 3), como também, a quantidade absoluta de cálcio absorvido (Tabela 2). Resultados que fazem supor que o transporte de cálcio ocorreu através de dois processos, ativo, saturável e passivo, concentração dependente. Essa afirmativa torna-se mais consistente quando se observam as taxas de absorção nos niveis compreendidos entre 90 e $150 \mathrm{ugCa} / \mathrm{ml}$ em que os respectivos valores de taxas de absorção não sofrem grandes alterações, região onde estaria ocorrendo o transporte máximo saturado pelo processo ativo.

Se o transporte de cálcio estivesse ocorrendo somente pelo processo ativo, a absorção, após ter atingido saturação máxima, permaneceria constante, já que a quantidade de transportadores existentes na membrana das células do intestino delgado é constante. Portanto, se a taxa de absorção de cálcio aumentou é porque a absorção de cálcio ocorreu, também, através do processo passivo, que é concentração dependente.

Segundo TOVERUD \& DOSTAL (1986), na equação de regressão linear $Y=A+B x, Y$ representa a quantidade de cálcio absorvido do lúmen intestinal para o sangue num determinado valor de cálcio introduzido $x$. A intersecção A com a ordenada é uma estimativa de transporte máximo de cálcio pelo processo ativo e a inclinação B representa a contribuição do processo passivo.

A regressão linear da quantidade de cálcio absorvido dos diferentes alimentos resultou para:

$\begin{array}{ll}\text { leite } & Y=32,93+0,33 x \\ \text { casca de ovo } & Y=52,42+0,37 x \\ \mathrm{CaCl}_{2} & Y=8,62+0,29 x\end{array}$

Observa-se, portanto, que o maior transporte de cálcio pelo processo ativo ocorreu com a casca de ovo e o menor com o $\mathrm{CaCl}_{2}$. Em relação ao componente passivo, o resultado foi melhor para a casca de ovo e menor para - $\mathrm{CaCl}_{2}$.

A determinação dos parâmetros cinéticos Vmax e $\mathrm{Km}$ (Tabela 4) resultaram das equações das curvas que caracterizaram o transporte ativo, ou seja, através da regressão inversa ponderada, como descrito no material e métodos.

Observam-se que os maiores valores $\mathrm{Vmax}$ e $\mathrm{Km}$ ocorreram com o leite quando comparados com a casca de ovo ou $\mathrm{CaCl}_{2}$. 
A quantidade de cálcio absorvida pelo processo ativo foi maior para a casca de ovo; no entanto, a velocidade máxima de absorção foi maior para o leite, resultado esse que permite sugerir que, provavelmente, os fatores existentes no leite tenham de alguma forma afetado o valor de Vmax.

O Km está relacionado com a afinidade do cálcio ao transportador de maneira inversa, ou seja, quanto maior o valor de $\mathrm{Km}$ menor é a afinidade, e representa a concentração de cálcio quando o Vmax é igual a metade. Neste contexto, os resultados demonstram que a maior afinidade do cálcio ao transportador ocorreu com o $\mathrm{CaCl}_{2}$ e a menor com o leite.

É importante frisar que é difícil medir com precisão Vmax e $\mathrm{Km}$ de transporte ativo de cálcio "in vivo" (IRELAND \& FORDTRAN, 1973). Isto porque é impossivel medir diretamente o transporte passivo de cálcio, à medida que a concentração de cálcio é aumentada acima da concentração de cálcio plasmático.

Um dos métodos para estimar o componente passivo foi apresentado neste trabalho e a partir desses dados obtidos, o componente de transporte ativo foi derivado indiretamente. Devido a essa incerteza envolvendo o componente difusional, as constantes cinéticas derivadas dessa análise devem ser consideradas como aproximações.

Tanto isso é verdade que FAVUS (1985) afirma em seu trabalho que as características cinéticas dos transportadores poderão ser precisamente determinadas somente quando o processo de transporte for totalmente caracterizado no nivel molecular.

Além disso, as diferentes metodologias utilizadas para a determinação dos parâmetros cinéticos, como por exemplo, as técnicas da alça ligáda "in vivo" e "in situ", perfusão "in vivo", alças intestinais "in vitro", são fatores que contribuem para os diferentes valores de Vmax e $\mathrm{Km}$ encontrados em diferentes experimentos.
Como exemplo do que foi afirmado acima, temos que no trabalho de PANSU et al. (1981), frilizando-se do método de perfusão, com um meio de 0,2 a $3,4 \mathrm{mM}$ de cálcio, encontraram valores de $V \max$ igual a 23 umol$\mathrm{Cal} / \mathrm{g}$.h e $\mathrm{Km}$ de $1,05 \mathrm{mM}$. Por outro, IRELAND \& FORDTRAN (1973) encontraram os valores de $0,29 \mathrm{molCa} / 60$ para Vamx e $3,5 \mathrm{mM}$ para $\mathrm{Km}$, realizando a experiência no jejuno dos humanos pelo método de perfusão. Já os autores OMDAHL \& DELUCA (1973) encontraram os valores de 28 umolCa/h e $36^{\prime}$ umolCa para $V$ max e $\mathrm{Km}$, respectivamente, utilizando-se da técnica da alça ligada no duodeno de rato.

Tais resultados divergentes são devidos, também, às condições em que foram realizadas as experiências, como por exemplo, ratos tratados ou não com vitamina D, dietas de animal contendo ou não, baixo ou elevado teor de cálcio, idade dos animais utilizados, entre outros.

Os valores de $\mathrm{Km}$ abaixo de $0,2 \mathrm{mM}$ são consistentes com um processo de transporte epitelial de significância fisiológica quando a concentração de cálcio luminal é baixo. É o que ocorreu com os resultados por nós obtidos com o maior valor de $\mathrm{Km}$ igual a $1,63 \mathrm{mM}$ para o leite, $0,35 \mathrm{mM}$ para a casca de ovo e 0,09 $\mathrm{mM}$ para $\mathrm{CaCl}_{2}$. Com relação aos valores de Vmax por nós observados para os diferentes alimentos não diferem muito dos valores encontrados na literatura, levando-se em consideração as ressalvas citadas anteriormente.

\section{4- CONCLUSŌES}

Os parâmetros absortivos determinados, quantidade total de cálcio absorvido, porcentagem de absorção e taxa de absorção resultaram melhores na casca de ovo quando comparados com o leite ou $\mathrm{CaCl}_{2}$. Entretanto, em relação aos parâmetros cinéticos $\mathrm{Vmax}$ e $\mathrm{Km}$, o leite foi o melhor.

\section{TABELA 1 - TEOR DE CÅLCIO DETERMINADO NO CONTEŮDO INTESTINAL DE RATOS TRATADOS COM SOLUÇÖES DE LEITE, CASCA DE OVO E CaCl 2 EM ug}
LEITE
concentração de soluções de leite (ugCa/ml)

$\begin{array}{rrrr} & 66,25 & 75,63 & 91,86 \\ * & 146,36 & 112,80 & 137,63\end{array}$
116,86
188,60
165,00
251,93
490,25
612,70

\begin{tabular}{|c|c|c|c|c|c|c|}
\hline $\begin{array}{l}\text { CASCA DE } \\
\text { OVO }\end{array}$ & \multicolumn{6}{|c|}{ concentração de soluções de casca de ovo (ugCa/mi) } \\
\hline & 61,25 & 74,00 & 90,00 & 118,75 & 181,00 & 419,00 \\
\hline * & 100,58 & 116,10 & 130,63 & 162,65 & 251,99 & 513,00 \\
\hline $\mathrm{CaCl}_{2}$ & \multicolumn{6}{|c|}{ concentração de soluções de $\mathrm{CaCl}_{2}(\mathrm{ugCa} / \mathrm{ml})$} \\
\hline & 48,00 & 74,00 & 105,00 & 134,00 & 162,00 & 204,00 \\
\hline * & 129,28 & 149,69 . & 190,33 & 241,33 & 282,83 & 349,39 \\
\hline
\end{tabular}

* $n=10$ ( $n$ - de ratos tratados para cada uma das concentrações) 
TABELA 2 - QUANTIDADE TOTAL DE CÅlCIO ABSORYIDO DO LEITE, DA CASCA DE OVO E DE CaCl2 EM 30 MINUTOS EM ug

\begin{tabular}{|c|c|c|c|c|c|c|}
\hline \multirow[t]{3}{*}{ LEITE } & \multicolumn{6}{|c|}{ concentração de soluções de leite (ugCa/ml) } \\
\hline & 66,25 & 75,63 & 91,86 & 116,86 & 165,00 & 490,00 \\
\hline & 46,90 & 99,22 & 106,85 & 105,88 & 138,83 & 428,06 \\
\hline \multirow{2}{*}{$\begin{array}{l}\text { CASCA DE } \\
\text { OVO }\end{array}$} & \multicolumn{6}{|c|}{ concentração de soluçöes de casca de ovo (ugCa/ml) } \\
\hline & 61,25 & 74,00 & 90,00 & 118,75 & 181,00 & 410,00 \\
\hline * & 94,30 & 104,28 & 121,75 & 147,23 & 182,39 & 379,38 \\
\hline \multirow[t]{3}{*}{$\mathrm{CaCl}_{2}$} & \multicolumn{6}{|c|}{ concentração de soluçōes de $\mathrm{CaCl}_{2}(\mathrm{ugCa} / \mathrm{ml})$} \\
\hline & 48,00 & 74,00 & 105,00 & 134,00 & 162,00 & 204,00 \\
\hline & 26,72 & 58,31 & 79,67 & 86,67 & 101,17 & 118,61 \\
\hline
\end{tabular}

* $n=10$ ( $n$ - de ratos tratados para cada uma das concentrações)

TABELA 3 - PORCENTAGEM DE ABSORÇĀO DOS ALIMENTOS EM DIFERENTES NIVEIS DE CONCENTRAÇĀO

\begin{tabular}{lcc}
\hline $\begin{array}{l}\text { LEITE } \\
\text { conc. ugCa/mi }\end{array}$ & \% abs. & $\begin{array}{l}\text { taxa abs. } \\
\text { ugCa/min }\end{array}$ \\
\hline 66,25 & 35,40 & 1,56 \\
75,63 & 65,60 & 3,30 \\
91,86 & 58,16 & 3,56 \\
116,86 & 45,30 & 3,52 \\
165,00 & 42,10 & 4,63 \\
490,25 & 43,66 & 14,26 \\
\hline
\end{tabular}

\section{CASCA DE OVO} conc. ug $(a / m)$ )

761,25
74,00
90,00
118,75
181,00

410,00

\section{$\mathrm{CaCl}_{2}$}

conc. ug $\mathrm{Ca} / \mathrm{ml}$

$$
\text { s }
$$

48,00

74,00

105,00

134,00

162,00

204,00
$\%$ abs.

76,98

70,46

67,64

62,00

50,38

46,26

\section{taxa abs.}

ugCa/min

3,14

3,48

4,06

4,90

6,08

12,65 
TABELA 4 - PARÂMETROS CINÉTICOS Vmax

Componentes do transporte ativo

$V_{\max } \mathrm{Km}$

(ugCa/min)
(ugCa/mi)

ou

$\mathrm{mM}$

Componentes do transporte passivo

\begin{tabular}{lrrlll}
\hline leite & $25,64+0,05$ & $65,08+0,33$ & 1,63 & $0,33+0,07$ & 0,81 \\
casca de ovo & $12,82+0,02$ & $13,96+0,27$ & 0,35 & $0,37+0,04$ & 0,83 \\
$\mathrm{CaCl}_{2}$ & $1,38+0,24$ & $3,40+1,64$ & 0,09 & $0,29+0,05$ & 0,87 \\
\hline
\end{tabular}

OGUIDO, Alice Katsuko; DE ANGELIS, Rebeca Carlota. Kinetics of intestinal calcium absorption from milk and egg shell in the rats. Semina: Ci. Agr., Londrina, v. 16, n. 1, p. 7-13, Mar. 1995.

ABSTRACT:This study was conducted to investigate the kinetics of calcium absorption from milk and egg shell "in situ" and "in vivo" loop preparation. The kinetic parameters were determined by the least squares method, by the linear regression and by the inverse ponderate regression. The determined absorptive parameters were considered better in the egg shell when compared with milk or $\mathrm{CaCl}_{2}$, but the kinetic parameters were considered better in the milk.

KEY-WORDS: Calcium absorption; kinetics; milk; egg shell.

\section{REFERENCIAS BIBLIOGRÁFICAS}

ALLEN, L.H. Calcium bioavailability and absorption: a review. Am. J. Clin. Nutr., vo 35, p. 783-808, 1982.

ARMBRECHT, H.J.; ZENSER, T.V。; DAVIS, B.B. Effect of vitamin $D$ metabolites on intestinal calcium absorption and calcium binding protein in young and adult rats. Endocrinology, v. 106, p. 469-75, 1980.

BRONNER, F; PANSU, D。; W.D. An analysis of intestinal calcium transport across the rat intestine. Am. J. Physiolo, v. 250, G.561-9, 1986.

BUCLON, M.; ROBINSON, J.W.L。; SEPULVEDA, F.V. Lack of effect of intracellular sodium on phenylalanine and B-methyl-glucoside in fiux into guinea-pig erythrocyte. J. Physiolo, v. 75, p. 571-9, 1979.

CLELAND, W.W. The statistical analysis of enzyme kinetic data. Adv. Enzymolo, v. 29, p. 1.32, 1967.

FA V US, M.J. Factor that influence absorption and secretion of calcium in the small intestine and colon. Am. J. Physiolo v. 248, G147-57, 1985 .

HEANEY, R.P。; SAVILLE, P.D。; RECHER, R.R. Cakium absorption as a function of calcium intake. J. Lab. Clin. Med., v. 85, p. 881-90, 1975.

IRELAND, P.; FORDTRAN, J.S. Effect of dietary calcium and age on jejunal calsium absorption in humans studied by intestinal perfusion. J. Clin。 Invest, v. 52, p. 2672-81, 1973.

LOTT, WoLa; NERY, J.Po; GALLO, JaRo; MEDCALF, J.C. A ఓ́cnica de análise foliar aplicada ao cafeoiro. New Yorks IBEC, Research Institute, 1956. (Bolatim IRI, 9)
MALM, O.J. Calcium requirement and adaptation in adult men. Scand. J. Clin. Lab. Ivesto, v. 10, p. 1, 1958. Supl. 36

MARS HALL, D.H. Calcium and phosphate kinetics in calcium, phosphate and magnesium metabolism. B.E.C. Nordin London: Churchill, 1976. 257p.

MCBEAN, L.D.; SPECKMANN, E.W. A recognition of the interrelationship of calcium with various dietary components. Am. J. Clin. Nutro, v. 6, p. 603-9, 1974.

OMDAHL, J.L_; DELUCA, H.F. Regulation of vitamin D motabolism and function. Physiol. Revo, v. 53, p. 327-72, 1973.

PANSU, D。; DUFLOS, Co; BELLATON, Co; BRONNER, F. Solubility and intestinal transit time limit calcium absorption in rats. J. Nutro, v. 123, p. 1396-1404, 1993.

PANSU, Do; BELLATON, Coi ROCHE, Co; BRONNER F。 $_{0}$ Duodenal and ileal calcium absorption in rat and effects of vitamin D. Am. J. Phisiolo, vo 244, G.695-700, 1983.

Ca intake on saturable and nonsaturable components of duodenal Ca transport. Am, Jo Phisiolo, v. 240, G.32-7, 1981.

PANSU, Do; CHAPUYS, M.C.; MILANI, Mo; BELLATON, C. Transepithelial calcium transport enchanced by xylose and glicose in the rat jejunal ligated loop. Calcif Tissue Res., v. 21, p. 45-52, 1976 .

PAPWORTH D.G. $_{0}$ PATRICK, G. The kinetics of imilux of calcium and strontium into rat intestine in vituro. J. Physiolo, v. 210, p. 999-1020, 1970 . 
RECHER, R.R.; BAMMI, A.; BAYER-LUX, M.J.; HEANEY, R.P. Calcium absorbability from milk products, an imitation milk and calcium carbonate. Am. J. Clin. Nut., v. 47, p. 93-5, 1988.

ROSEMBERG, I.H.; SOLOMONS, N.W. Biological availability of minerals and trace elements: a nutritional overview. Am. J. Clin. Nut., v. 35, p. 781-2, 1982.

TOVERUD, S.U.; DOSTAL, L.A. Calcium absorption during development: experimental studies of the rat small intestine. J. Pediat. Gastoenter. Nut, v. 5, p. 688-95, 1986.

WALLING, M.W.; ROTHMAN.S.S. Phosphate independent, carrier active transport by rat intestine. Am. J. Physiol., v. 217 , p. $1144-8.1969$.
W ASS ERMA N, R.H. Intestinal absorption of calcium and phosphorus. Federation Proc, v. 40, p. 68-72. 1981.

W ASS ERMA N, R.H.; FULLMER, C.S. Calcium transport proteins, calcium absorption and vitamin D. Ann. Rev. Physiol., v. 45, p. 375-90, 1983.

WASSERMAN, R.H.; TAYLOR, A.N. Some aspects of intestinal absorption of calcium with special reference to vitamin D. In: COMAR, C.L.; BRONNER, F. Mineral metabolism. An advanced treatise. New York: Academic Press, 1969. v. 3, p. 321-403. 